Prawo Kanoniczne

59 (2016) $\mathrm{nr} 4$

\author{
JESÚS MIÑAMBRES \\ Pontificia Università della Santa Croce, Roma
}

\title{
AUTONOMIA E RESPONSABILITÀ NELLA AMMINISTRAZIONE DELLE RISORSE DELLA CHIESA*
}

Sommario: 1. Autonomia gestionale. - 2. Autonomia come principio di organizzazione per la gestione dei beni della Chiesa. - 3. La gestione delle risorse messe a disposizione dell'organizzazione. -4 . Responsabilità giuridica degli enti e degli amministratori. -5 . Responsabilità giuridica dei singoli fedeli. -6 . La riparazione dei danni. - Conclusioni.

\section{Autonomia gestionale}

L'autonomia è uno di quei concetti polisemici, presi dalla considerazione filosofica della realtà, che viene adoperato nel mondo giuridico con sfumature diverse a seconda degli ambiti di applicazione ${ }^{1}$. Nel contesto giuridico canonico in cui ci muoviamo in queste pagine ${ }^{2}$,

\footnotetext{
* Traduzione italiana del contributo preparato in occasione del venticinquesimo anniversario della Facoltà di Diritto canonico della Universidad Católica Argentina.

${ }^{1}$ Cfr. A. Viana, La norma estatutaria y la autonomía de los entes en la Iglesia, in: J. Canosa (cur.), I principi per la revisione del Codice di Diritto canonico. La ricezione giuridica del Concilio Vaticano II, Milano 2000, p. 271.

${ }^{2}$ Come si vedrà, non ci interessano qui alcuni dei sensi in cui è adoperata la nozione di autonomia nell'ordinamento canonico, come l'autonomia delle realtà temporali (cfr. J.T. MARTín DE AGAR, Autonomía de las realidades temporales, in: Diccionario General de Derecho Canónico, vol. I, Cizur Menor 2012, p. 576-578), riconosciuta dal Concilio Vaticano II, oppure l'autonomia privata delle persone (cfr. E Molano, Autonomía privada, in: Diccionario General de Derecho Canónico, vol. I, cit., p. 582-588).
} 
l'autonomia riguarda soprattutto la capacità di un ente di darsi norme, di generare atti che costringano a determinati comportamenti, sia che si tratti di leggi in senso proprio (cfr. can. 8), sia che si adoperino norme amministrative (cfr. can. 30, can. 31, ecc.) $)^{3}$ o statutarie ${ }^{4}$.

Il Sinodo dei Vescovi del 1967 aveva incluso tra i principi che dovevano guidare la redazione del Codice di Diritto canonico, rinnovato con le indicazioni venute dal Concilio Vaticano II, quello dell'autonomia, come corollario della sussidiarietà: «(...) si propugnano anche la convenienza e la necessità di provvedere all'utilità dei singoli istituti, in modo speciale, attraverso i diritti particolari e una sana autonomia della potestà esecutiva particolare (...).» ${ }^{5}$. In questo senso, l'autonomia non riguarda soltanto l'esercizio della potestà legislativa ("i diritti particolari"), ma anche di quella esecutiva.

Nell'uso corrente, è frequente adoperare l'autonomia per descrivere la possibilità di indirizzare la propria condotta verso delle finalità prestabilite, senza costrizioni esterne. Ed è in questo senso che il principio ci interessa per parlare dell'amministrazione delle risorse e in particolare dei beni ecclesiastici degli enti canonici. In effetti, la regola principale che guida ogni atto di amministrazione dei beni della Chiesa, senza la quale l'atto non avrebbe senso, è quella che potrebbe essere denominata "teleologica", che richiede la finalizzazione dell'attività sui beni agli scopi istituzionali riassunti nel secondo paragrafo del can. 1254: «I fini propri sono principalmente: ordinare il culto divino, provvedere ad un onesto sostentamento del clero e degli altri ministri, esercitare opere di apostolato sacro e di carità, specialmente a servizio dei poveri». Perciò, la capacità di determinare

\footnotetext{
${ }^{3}$ Cfr. J.I. Arrieta, Diritto dell'organizzazione ecclesiastica, Milano 1997, p. 97.

${ }^{4}$ Cfr. A Viana, La norma estatutaria y la autonomía de los entes en la Iglesia, cit., p. 271-301.

${ }^{5}$ Prefazione al CIC. Il testo latino originale dell'enunciazione del principio può essere consultato in «Communicationes» 2 (1969) 81.

${ }^{6} \mathrm{Ci}$ siamo occupati di questo argomento in un contributo dal titolo Principi di organizzazione del governo patrimoniale delle entità ecclesiastiche, pubblicato in: G. Boni, E. Camassa, P. Cavana, P. Lillo, V. Turchi (cur.), "Recte sapere". Studi in onore di Giuseppe Dalla Torre, Torino 2014, vol. I, p. 454-466.
} 
lo scopo dei propri atti di amministrazione, l'esercizio del principio di autonomia nella gestione delle risorse dell'ente, diventa fondamentale, quasi un presupposto dell'amministrazione.

Il legislatore canonico ha espresso il criterio che determina il modo dell'amministrazione dei beni ecclesiastici con la frase tradizionale in questa materia: «con la diligenza di un buon padre di famiglia» (can. $1284 \$ 1)$. Tale diligenza richiede autonomia, capacità cioè di adeguare i mezzi alle finalità: ogni padre di famiglia fa questo e ogni amministratore di beni ecclesiastici deve essere messo nelle condizioni di farlo, altrimenti sarebbe ingiusto chiedergli un comportamento che non potrebbe tenere. In termini più tecnici, la gestione delle risorse in modo responsabile richiede "autonomia gestionale", cioè la capacità di "prendere decisioni in modo discrezionale e indipendente da fattori esterni ${ }^{7}$, senza però rinunciare al "rapporto con l'ambiente" circostante, cioè negli enti ecclesiastici senza rinunciare a contribuire alla costruzione della comunione.

Questa percezione della necessità di autonomia nella gestione, se si preferisce nell'amministrazione, comporta che l'amministratore in senso proprio di una persona giuridica canonica pubblica è chi la regge immediatamente, come ricorda il can. $1279 \$ 1$, e non chi ricopre altre funzioni nell'ente, anche quando tali funzioni riguardino espressamente la gestione delle risorse ${ }^{8}$. Così si mette l'amministratore

\footnotetext{
${ }^{7}$ L. Hinna, Economia delle aziende pubbliche e non profit. L'accountability nella PA, in: https://www.google.it/url?sa=t\&rct=j\&q=\&esrc=s\&source $=\mathrm{w}$ eb\&cd=1\&ved=0ahUKEwiFkLWR29HNAhVFrRoKHdFoD0IQFggcMA A\&url=http\%3A\%2F\%2Fwww.uniroma2.it\%2Fdidattica\%2FEc.aziende. pubbliche\%2Fdeposito\%2F10_Juris_2011_2012.ppt\&usg=AFQjCNHHQdSQ-MUK9k2uES8_yXcdoHZOag\&bvm=bv.125801520,d.d2s\&cad=rja.

${ }^{8}$ In questo senso, siamo sempre più convinti che l'economo diocesano non possa essere ritenuto "amministratore" dei beni della diocesi: l'amministratore è necessariamente il vescovo, che deve svolgere il suo compito come un buon padre di famiglia e che risponde per le inadempienze, le mancanze di diligenza, gli atti invalidi o illegittimi, ecc. L'economo, invece, è un collaboratore del vescovo, che lo aiuta con la sua perizia e con il suo lavoro, ma che in senso proprio non amministra il patrimonio della diocesi. Più avanti vedremo le ricadute che questo modo di impostare l'organizzazione diocesana ha sull'individuazione delle responsabilità.
} 
nel posto strutturale che gli spetta nell'organizzazione ecclesiastica e gli si attribuiscono i mezzi necessari per portare avanti il suo compito come "un buon padre di famiglia", con la necessaria autonomia e responsabilità. L'amministratore è responsabile del conseguimento delle finalità della persona giuridica. Anche se si avvale della collaborazione di altre persone (tecnici, consulenti, ecc.), alla fine è l'amministratore chi deve prendere le decisioni e, quindi, chi deve rispondere per le loro conseguenze. Perciò, senza entrare adesso nella complessa vicenda della classificazione della potestà, che esula da queste considerazioni, bisogna comunque ribadire che l'amministratore è la figura sulla quale ricade la responsabilità per gli atti giuridici posti dall'ente ecclesiastico. E per lo stesso motivo, l'autonomia gestionale intesa nel senso prima descritto è condizione di un'amministrazione responsabile.

L'autonomia e la responsabilità che ci interessano di più in questo studio riguardano però, in primo luogo, l'ente e non l'amministratore o un qualsiasi altro organo o rappresentante dell'ente. In questo senso, chi è autonomo e chi risponde per gli atti compiuti in suo nome è la persona giuridica stessa (cfr. can. $1281 \$ 3$ ), ma avendo bisogno di organi o rappresentanti per agire, entrambe le caratteristiche dell'ente si riflettono anche sulla persona fisica che amministra le sue risorse,

Per ulteriori delucidazioni e indicazioni bibliografiche, cfr. V. DE PAolis, I beni temporali della Chiesa, Bologna 1995, p. 150-151; F.R. AzNAR GIL, El ecónomo diocesano, in: AA.VV., La Curia diocesana. La función administrativa, Salamanca 2001, p. 299-337; J.A. Di Nicco, El ecónomo diocesano. Precisiones acerca de este oficio eclesiástico: propuesta sobre el parágrafo tercero del canon 494, Buenos Aires 2012; J. MiÑAmbres, Funzione amministrativa e amministrazione dei beni ecclesiastici, in: J. Wrocenski-M. Stoklosa (cur.), La funzione amministrativa nell'ordinamento canonico. XIV Congresso internazionale di Diritto canonico, Varsavia, 14-18 settembre 2011, Warszawa 2012 (vol. 2), p. 963-972; H. PreE, Der Diözesanökonom, sein rechtliches Verhältnis zum Diözesanbischof und seine Rechtsstellung in der Bischöflichen Kurie, Archiv für katholisches Kirchenrecht 182 (2013) 24-43; J. MiñAmbres, La responsabilità canonica degli amministratori dei beni della Chiesa, Ius Ecclesiae 27 (2015) 577-594; ID., Corresponsabilità e amministrazione dei beni della Chiesa, in: F. Lozupone (cur.), Corresponsabilità e trasparenza nell'amministrazione dei beni della Chiesa, Ariccia 2015, p. 81-100. 
sia come autonomia di gestione, sia come responsabilità per gli atti posti nell'esercizio di tale amministrazione. Inoltre, l'ordinamento canonico ha sempre collegato la responsabilità per le conseguenze di un atto all'imputabilità dell'atto stesso ad un soggetto fisico ${ }^{9}$, in modo tale da rendere difficile la prospettazione di una "responsabilità oggettiva" in diritto canonico.

L'autonomia degli enti ecclesiastici all'interno della comunione della Chiesa è una caratteristica che potremmo denominare "problematica”. È problematica soprattutto perché, in alcuni casi, riflette esigenze di tipo teologico che poggiano direttamente sul "mistero" della Chiesa; si pensi al peculiare rapporto che intercorre tra Chiesa universale e Chiese particolari, che rende l'autonomia degli enti che le esprimono nel mondo del Diritto una novità nei confronti delle esperienze abituali di diritto costituzionale e di diritto pubblico. Il rapporto di "immedesimazione" che fa presenti le Chiese particolari nella Chiesa universale e la Chiesa universale nelle Chiese particolari è unico nell'esperienza delle organizzazioni sociali e soltanto si spiega e si sostiene in una visione di fede ${ }^{10}$. Tuttavia, una configurazione del principio di autonomia che non rispettasse queste peculiarità essenziali alla Chiesa non potrebbe essere applicata al suo interno.

\section{Autonomia come principio di organizzazione per la gestione dei beni della Chiesa}

Nel descrivere l'autonomia degli enti e dei loro amministratori abbiamo già fatto riferimento a quello che potrebbe essere denominato il principio teleologico, che dà il criterio legittimante per «acquistare, possedere, amministrare e alienare i beni temporali» (can. $1254 \$ 1$ ) e, quindi, per ogni singolo atto di gestione.

\footnotetext{
${ }^{9}$ Scrive Baura: «(...) nella determinazione delle conseguenze giuridiche è decisivo l'intervento o meno della volontà (libera) umana. La presenza della libertà umana (...) implica che un determinato atto sia attribuibile al suo autore perché suo: egli è il responsabile della sua azione e dei suoi effetti» (E. BAura, Parte generale del Diritto canonico. Diritto e sistema normativo, Roma 2013, p. 62-63).

${ }^{10}$ Spiega in poche pagine queste peculiarità, C.J. ErRÁzURIz M., Corso fondamentale sul Diritto nella Chiesa, I, Milano 2009, p. 296-302.
} 
Abbiamo fatto anche riferimento al principio di sussidiarietà, richiamato dal legislatore nella Prefazione del Codice di Diritto canonico ${ }^{11}$ come uno dei principi espressi dal Sinodo dei Vescovi per dirigere la revisione legislativa della Chiesa. La peculiarità del principio di sussidiarietà nell'organizzazione ecclesiastica ${ }^{12}$ proviene dal dato presupposto nell'enunciazione del principio fatta nel testo citato, e cioè dal fatto che «l'ufficio dei Vescovi con le potestà annesse è di diritto divino». È vero che vi è un vincolo gerarchico con il capo del Collegio dei Vescovi e con il Collegio stesso, in mancanza del quale si perde la comunione e ci si pone in una situazione di impossibilità di esercizio delle abilitazioni ricevute nell'ordinazione, ma è anche vero che tale vincolo gerarchico non esprime una dipendenza in senso proprio dall'autorità gerarchica superiore, giacché le potestà annesse all'ufficio sono di diritto divino ${ }^{13}$.

Queste premesse caratterizzano l'agire del principio di sussidiarietà nell'organizzazione ecclesiastica, in cui vi è l'interazione fra enti non rigidamente subordinati ma integrati in una comunione che poggia sull'attribuzione sacramentale di abilitazione al governo della comunità. Per quanto riguarda l'organizzazione preposta all'amministrazione dei beni temporali della Chiesa ${ }^{14}$, il principio

${ }^{11} \ll 5^{\circ}$ Probe attendatur ad principium (...) subsidiarietatis (...), in Ecclesia eo vel magis applicandum, quod officium episcoporum cum potestatibus adnexis est iuris divini. Hoc principio (...) convenientia etiam et necessitas propugnantur providendi utilitati praesertim singulorum institutorum per iura particularia et per sanam autonomiam potestatis exsecutivae particularis illis agnitam » (Praefatio al CIC).

${ }^{12}$ Cfr. C. CARdia, La rilevanza costituzionale del principio di sussidiarietà nella Chiesa, in: J. Canosa (cur.), I principi per la revisione del Codice di Diritto canonico. La ricezione giuridica del Concilio Vaticano II, Milano 2000, p. 233-270.

${ }^{13}$ Le difficoltà e le peculiarità che scaturiscono dal rapporto tra il sacramento dell'ordine e l'esercizio della potestà nella Chiesa sono state oggetto di molti studi e non possono essere da noi affrontate in questa sede. Per alcuni spunti e riferimenti bibliografici, cfr. J. Hervada, Diritto costituzionale canonico, Milano 1989, p. 231 ss.; J. I. Arrieta, Diritto dell'organizzazione ecclesiastica, Milano 1997, p. 37 ss.; C. J. Errázuriz, Corso fondamentale sul Diritto nella Chiesa. I. cit., p. 302 ss.

${ }^{14}$ Cfr. V. De Paolis, I beni temporali della Chiesa. Canoni preliminari (cann. 12541258) e due questioni fondamentali, in: AA. VV., I beni temporali della Chiesa, 
di sussidiarietà richiede che ogni ente sia messo nelle condizioni di gestire i propri beni per l'utilità generale della Chiesa, evitando di coinvolgere enti "superiori" che rispondano per l'operato di quelli ad essi sottoposti, e viceversa, che i superiori debbano rispettare l'autonomia degli enti inferiori.

Dall'autonomia e la sussidiarietà sorgono anche altri "modi" di organizzare che spesso assumono la caratterizzazione di principi (si pensi alla corresponsabilità ${ }^{15}$, alla partecipazione ${ }^{16}$, alla trasparenza ${ }^{17}$, ecc.) e che esprimono il "grande principio" dell'organizzazione ecclesiastica che è la comunione ${ }^{18}$.

Milano 1997, p. 20-29.

${ }^{15}$ Cfr., ad es., J. Miñambres, La "stewardship" (corresponsabilità) nella gestione dei beni temporali della Chiesa, Ius Ecclesiae 24 (2012) 277-292; ID., Corresponsabilità e amministrazione dei beni della Chiesa, in: F. Lozupone (a cura di), Corresponsabilità e trasparenza nell'amministrazione dei beni della Chiesa, Ariccia 2015, p. 81-100; M. Rivella, Buon governo e corresponsabilità, Ius Ecclesiae 24 (2012) 293-302; D. Zalbidea, Corresponsabilidad (stewardship) y Derecho Canónico, Ius Ecclesiae 24 (2012) 303-322.

${ }^{16}$ Il governo della comunità in generale, e in particolare la gestione dei suoi beni, esige una qualche partecipazione di tutti i suoi componenti. Cfr., ad es., P. Asolan, Corresponsabilità e partecipazione dei fedeli al "munus regendi Christi", Annales Theologici 28 (2014) 417-434; J.-P. Schouppe, Diritti fondamentali dei fedeli in rapporto alla partecipazione al governo dei beni temporali, Ius Ecclesiae 26 (2014) 397-413.

${ }^{17}$ Cfr., ad es., A. Granados, La comunicazione del progetto pastorale come via per stimolare la corresponsabilità ecclesiale, Annales Theologici 28 (2014) 153-165; G. DAмm Aсco, Trasparenza e onestà nell'amministrazione del beni ecclesiastici, in: F. Lozupone (a cura di), Corresponsabilità e trasparenza nell'amministrazione dei beni della Chiesa, Ariccia 2015, p. 43-64.

${ }^{18}$ È chiaro che l'elenco dei principi che guidano l'organizzazione ecclesiastica potrebbe essere allargato o ristretto dipendendo dalla prospettiva che vi si adotti volta per volta. Ad esempio, mons. Arrieta, studioso di questa materia, dedica un'epigrafe del suo manuale ai "Principi di organizzazione dei rapporti tra uffici ecclesiastici" ed elenca i seguenti: Principio di unità o di totalità, principio di deconcentrazione, principio di vicarietà, principio di competenza organica, principio di gerarchia organica, principio di direzione e principio di coordinamento (cfr. J.I. ARRIETA, Diritto dell'organizzazione ecclesiastica, Milano 1997, p. 156 ss.). 
In effetti, la comunione più che un principio di organizzazione è un modo di descrivere la realtà essenziale della stessa Chiesa ${ }^{19}$. Nel senso in cui può essere descritta come principio per l'organizzazione ecclesiastica, la comunione richiede che tale organizzazione riesca a mostrare visibilmente fra gli uomini il mistero della Chiesa. Compito non facile che, nella gestione delle risorse a disposizione per il raggiungimento degli scopi prefissati ai diversi enti, dovrà manifestarsi ad esempio nella degna celebrazione dei sacramenti (con i luoghi e i beni adeguati), in modo particolare l'Eucaristia ${ }^{20}$, e nel testimonio della carità, il "servizio della carità" secondo un'espressione cara a Benedetto XVI ${ }^{21}$. Perché la comunione che descrive l'essenza della Chiesa non può essere ridotta a puro "principio" di organizzazione, soprattutto se si pensa alle sue diverse dimensioni (orizzontale, comunione tra gli uomini, e verticale, comunione con Dio; visibile e invisibile $\mathrm{e}^{22}$, ecc.), che la rendono scopo e ispirazione, traguardo e permanente acquisizione, anelito e guida per l'azione... Quindi, il principio organizzativo della comunione si rivela anche lo scopo dell'organizzazione stessa, e perciò principio anche di azione, di attuazione delle finalità di tutti gli enti.

A sua volta, non occorre perdere di vista che la comunione non significa uniformità, ma piuttosto unità nella diversità; non si può essere in comunione con sé stessi, la comunione esige relazione. Perciò, nel contesto che adesso ci interessa, lo scopo finale della comunione viene raggiunto con gli apporti diversi dei singoli fedeli e di ciascun ente nella sua peculiarità. Ciò rende necessario che ognuno esprima il proprio contributo con personale responsabilità, in autonomia (autonomia dei fedeli nella gestione delle proprie vite e autonomia

${ }^{19}$ Cfr. Congregazione per la Dottrina della Fede, Communionis notio, 28 maggio 1992, AAS 85 (1993) 838-850, n. 1.

${ }^{20}$ Cfr. Congregazione per la Dottrina della Fede, Communionis notio, cit., n. 5 .

${ }^{21}$ Cfr. Benedetto XVI, Intima Ecclesiae natura, 11 novembre 2012, AAS 104 (2012) 996-1004.

${ }^{22}$ Cfr. Congregazione per la Dottrina della Fede, Communionis notio, cit., n. 3 e 4 . 
degli enti nel raggiungimento dei propri scopi) per arricchire la comunione nella diversità ${ }^{23}$.

\section{La gestione delle risorse messe a disposizione dell'organizzazione}

Una volta stabiliti i confini organizzatori della Chiesa istituzione, sia per esigenze di diritto divino, sia mediante lo sviluppo legislativo umano, le risorse destinate a costruire la comunione della Chiesa devono essere effettivamente ed efficacemente destinate al loro scopo. In questa prospettiva, il centro dell'attenzione diventa l'amministratore della persona giuridica e non più l'ente da lui amministrato, giacché come abbiamo ribadito, le persone giuridiche agiscono sempre attraverso organi o rappresentanti, normalmente composti da una singola persona fisica, e talvolta da più persone fisiche preposte all'espressione di una volontà comune.

Nel titolo di questo studio abbiamo voluto cambiare il termine tecnico "beni ecclesiastici" (can. $1257 \$ 1$ ) con il più generico "risorse", in un tentativo, forse poco riuscito, di includere nel concetto non soltanto la gestione dei beni temporali ma anche il governo delle persone. A nostro parere, è molto difficile, forse impossibile, separare la gestione dei beni da quella delle persone. Ci rendiamo conto che il termine "gestione" unito a "persona" produce una reazione quasi di ribrezzo, come se si cercasse la riduzione delle persone, nel nostro caso dei fedeli, a strumenti dell'organizzazione. Come è evidente, lo scopo non è questo, ma piuttosto sottolineare che la realizzazione della comunione, anche attraverso l'opera dell'organizzazione, richiede non soltanto la guida della comunità, ma anche l'impiego di beni materiali, o viceversa, non soltanto l'uso dei beni temporali quanto anche la guida della comunità. E, per quanto ora ci interessa, l'uso del termine risorse anziché beni può aiutare a non perdere di vista che quello che conta è che tutti i fedeli e tutte le cose convergano verso la realizzazione della comunione e della missione della Chiesa.

\footnotetext{
${ }^{23}$ Cfr. Cfr. Congregazione per la Dottrina della Fede, Communionis notio, cit., n. 7 e n. 15-16.
} 
Questa considerazione manifesta, a nostro parere, la complessità dell'amministrazione o gestione delle risorse della Chiesa per la comunione e può essere ritenuta la base dalla quale parte il legislatore per descrivere la responsabilità dell'amministratore come «la diligenza di un buon padre di famiglia» (can $1284 \$ 1$ ). Nell’immagine della "famiglia" già adoperata dal Concilio Vaticano II per descrivere la Chiesa $^{24}$, il ruolo della paternità e della maternità ${ }^{25}$ si indirizza verso il bene di tutta la famiglia; in modo analogo, appunto, il ruolo dell'amministratore nella Chiesa è rivolto alla costruzione della comunione. In tal senso, la prima responsabilità dell'amministratore è la ricerca della comunione, l'uso di tutti i mezzi a sua disposizione per costruire la comunione. In altre parole, l'amministratore dei beni ecclesiastici giustifica il proprio operato in quanto riesce a destinare le risorse agli scopi per i quali esse possono essere legittimamente acquisite, possedute, amministrate ed alienate, cioè quando rende presente nell'agire quello che abbiamo descritto come principio teleologico per configurare l'organizzazione della Chiesa, quando indirizza tutte le potenzialità verso il culto, il sostentamento del clero e l'esercizio della carità e dell'apostolato (cfr. can. $1254 \$ 2$ ).

Per compiere la sua funzione, quindi, l'amministratore deve ispirare la sua azione sulle risorse al raggiungimento della comunione, adoperando gli strumenti che l'ordinamento gli offre per riuscirci. Qui può essere utile rifarsi ad altri principi, questa volta non di organizzazione ma di azione amministrativa, che consentano di esprimere concretamente la responsabilità del buon padre di famiglia. Gli

\footnotetext{
${ }^{24}$ Cfr. Concilio Vaticano II, Cost. dogm. Lumen gentium, 6.

${ }^{25}$ Com'è noto, l'espressione "buon padre di famiglia" compare già nelle fonti del Diritto romano imperiale e di quello giustinaneo e non intende discriminazione fra i sessi, anche se il diritto di famiglia la comportava. Noi abbiamo aggiunto il riferimento alla maternità perché la misura della responsabilità nella Chiesa non distingue tra le funzioni proprie della paternità e quelle della maternità in una famiglia, le comprende tutte. Di fatti, la figura del buon padre di famiglia è stata assunta come "criterio tradizionale" della misura della responsabilità anche da molte legislazioni civili (cfr., ad es., art. 1176 del Codice civile italiano del 1942; art. 1094 Código civil español, ecc.).
} 
studiosi del Diritto amministrativo canonico hanno stilato diversi elenchi di principi guida sia per la conformazione degli atti amministrativi, sia per i ricorsi che su di essi possono fondarsi ${ }^{26}$. Tra i principi che reggono l'agire degli amministratori viene menzionato al primo posto il principio di legalità dell'azione amministrativa ${ }^{27}$ : l'amministrazione deve assoggettarsi alla legge generale, anche se ciò non è incompatibile con la dovuta discrezionalità dell'autorità nel governo delle risorse e nemmeno con l'eventuale impugnazione degli atti amministrativi singolari da parte dei fedeli.

La pubblicità dell'azione amministrativa e la necessità di accordare ai fedeli una qualche partecipazione al governo delle risorse, fra loro molto collegate, si presentano, anche esse, come principi da rispettare nell'agire amministrativo nella Chiesa: la prima in relazione alle procedure pubbliche seguite nella formazione dell'atto amministrativo, che devono poter essere giustificate in ogni momento (accountability); la seconda, corollario dell'adeguata configurazione organizzativa della comunione nella Chiesa, si traduce nel convocare alle riunioni nelle quali gli organi devono esprimere la loro volontà tutti coloro che ne hanno diritto ${ }^{28}$, ascoltare i diversi collegi o seguire il loro parere ${ }^{29}$, ecc.

\footnotetext{
${ }^{26}$ Particolarmente ordinato e riassuntivo in questo senso, e riportante anche indicazioni bibliografiche, è il lavoro di J. CANosA, I principi e le fasi del procedimento amministrativo nel Diritto canonico, Ius Ecclesiae 18 (2006) 551-577.

${ }^{27}$ Cfr. E. Labandeira, Trattato di Diritto amministrativo canonico, Milano 1994, p. 175 ss.; J. Miras, J. Canosa, E. Baura, Compendio di diritto amministrativo canonico, Roma 2007, p. 53 ss.

${ }^{28}$ Cfr., già prima del Codice, P. Moneta, Procedimento amministrativo e partecipazione dei fedeli alla funzione amministrativa, Ius Canonicum 14 (1974) 25-41.

${ }^{29}$ Il legislatore chiede agli organi nei quali si esprime la partecipazione di tutti i fedeli all'azione di governo dei pareri che possono essere vincolanti o meno (cfr., tra i tanti, J.I. Arrieta, Órganos de participación y corresponsabilidad en la Iglesia diocesana, Ius Canonicum 34 (1994) 553-593; V, DE PAOLIs, Il Consiglio parrocchiale per gli affari economici e i beni patrimoniali della parrocchia, in: AA. VV., La parrocchia, Città del Vaticano 1997, p. 267-288; F. Coccopalmerio, La natura della consultività ecclesiale, in: M. Rivella (ed.), Partecipazione e corresponsabilità nella Chiesa. I consigli diocesani e parrocchiali, Milano 2000, p. 23-32; M. Rivella, Consigliare nella Chiesa in ambito economico, Quaderni di diritto ecclesiale 25 (2012)
} 
Per la produzione di atti amministrativi che riescano a costruire la comunione della Chiesa risulta molto interessante quello che Canosa chiama "principio di motivazione", vale a dire «l'obbligo di esporre i motivi della decisione ${ }^{30}$. Il principio stimola l'amministratore ad agire con la necessaria trasparenza e nel rispetto delle procedure stabilite, permettendo anche alle persone coinvolte nella decisione di presentare le loro eventuali rimostranze con maggiore conoscenza dei beni in gioco. Logicamente, tutta l'azione amministrativa va esercitata sotto la guida della virtù della prudenza che, nelle diverse circostanze, esprime esigenze di motivazione diverse: non sempre si potranno esprimere esplicitamente tutti i motivi di una scelta, ma sempre bisognerà giustificare giuridicamente l'intervento amministrativo, dare nell'atto stesso una spiegazione, almeno sommaria, del perché della decisione in esso espressa ${ }^{31}$.

Nell'agire degli amministratori è necessario anche attenersi ad un'adeguata formalità dell'atto, che nel caso della gestione delle risorse si sostanzia spesso in rapporti di tipo contrattuale e perciò deve seguire le norme "civili" vigenti, come stabilito per i contratti e i pagamenti dal can. 1290 o per l'accettazione dei testamenti e dei legati dal can. $1299 \$ 2$. Ma anche nelle occasioni in cui non si agisca mediante contratto e non si stia provvedendo a pagamenti, l'atto

390-399; ecc.). In materia di amministrazione dei beni temporali sono chiamati a partecipare soprattutto i consigli per gli affari economici, a tutti i livelli, e poi anche, nella diocesi, il collegio dei consultori e, in qualche occasione, il consiglio presbiterale (ad es., al can. 1263, per l'imposizione dei tributi diocesani), e negli istituti di vita consacrata, il consiglio del superiore. Per le esigenze normative generali riguardanti l'agire dei collegi, cfr. can. 127.

${ }^{30}$ J. CANosa, I principi..., cit., p. 558.

${ }^{31}$ Può aiutare a comprendere i limiti prudenziali della trasparenza nella produzione degli atti amministrativi il paragone fatto dal Concilio Vaticano II e già riportato nel testo tra Chiesa e famiglia: tutti in famiglia devono conoscere le decisioni che li riguardano e tutti devono essere in grado di comprenderne le motivazioni, ma non deve essere spiegato "tutto" a tutti. Comunque, non sarebbe giusto imporre una condotta per forza, senza alcuna motivazione. 
di gestione deve esprimere la propria natura in modo formalmente chiaro, presentandosi come atto di amministrazione ${ }^{32}$.

La gestione delle risorse ecclesiastiche deve essere tempestiva e ciò richiede che l'amministratore agisca con celerità, soprattutto quando l'azione dell'autorità è stata in qualche modo richiesta dagli amministrati. In riferimento all'ufficio dei Vescovi, il Direttorio per il ministero pastorale dei Vescovi Apostolorum successores ${ }^{33}$ ricorda che il «rapido disbrigo delle questioni è norma di ordinata amministrazione e anche di giustizia verso i fedeli» (n. 69).

L'ordinato esercizio del governo in qualunque sede richiede che gli atti amministrativi godano di stabilità, nel senso che una volta emanati sono vincolanti per tutti gli amministrati, senza perciò impedire l'esercizio del diritto al ricorso (richiesta di revoca o modifica dell'atto; ricorso gerarchico; esercizio dell'azione contenziosa). Nella gestione delle risorse, la stabilità e il diritto al ricorso sono anche garanzia di buon governo delle stesse.

Lamministrazione delle risorse ecclesiali si avvale quindi delle modalità di attuazione della potestà amministrativa in generale. Certamente, potrebbero essere aggiunte alcune caratteristiche tipiche specifiche della gestione di risorse materiali, che in qualche modo possono essere fatte risalire all'una o all'altra delle esigenze generali di esercizio del governo, ma che trovano espressioni peculiari nell'amministrazione dei beni. Si pensi, in particolare, a quella specifica forma della responsabilità che si è soliti chiamare accountability, alla trasparenza, ecc.

Non si può non richiamare, in fine, la modalità richiesta espressamente dal legislatore per l'amministrazione dei beni ecclesiastici nel can. 1282: «Tutti coloro (...) che (...) hanno parte nell'amministrazione

\footnotetext{
${ }^{32}$ Talvolta gli atti amministrativi sono contenuti in scritti di altro genere, come lettere o risposte di vario tipo; anche in questi casi, l'adeguata formalità richiede che sia espresso il carattere e il valore dell'atto, soprattutto al fine di permettere la sua impugnazione da parte del ricevente.

${ }^{33}$ Congregazione Per i Vescovi, Direttorio per il ministero pastorale dei Vescovi "Apostolorum successors", 22 febbraio 2004, Città del Vaticano 2004.
} 
dei beni ecclesiastici, sono tenuti ad adempiere i loro compiti in nome della Chiesa (...) ». Anche se è difficile precisare in ogni fattispecie cosa significhi agire nomine Ecclesiae, è chiaro che la prescrizione legale ribadisce la "pubblicità" dell'amministrazione dei beni ecclesiastici, nel senso di essere destinati al raggiungimento delle finalità istituzionali della Chiesa. Questa condizione di pubblicità dei beni ecclesiastici, che il can. 1257 descrive come appartenenti appunto a persone giuridiche pubbliche, si trasferisce anche alla gestione degli stessi. Qui radica la differenza essenziale di regime giuridico legale tra i beni ecclesiastici e quelli che appartengono ad altri soggetti nella Chiesa, in particolare quelli che sono nella titolarità di persone giuridiche canoniche private.

\section{Responsabilità giuridica degli enti e degli amministratori}

Come abbiamo più volte accennato, la responsabilità nella gestione dei beni ecclesiastici trae origine dai rapporti che sorgono fra i diversi soggetti impegnati: la persona giuridica titolare dei beni, il suo amministratore, assistito dal consiglio per gli affari economici, e l'economo, quando previsto. Si avvera una prima relazione tra la persona giuridica pubblica titolare dei beni (cfr. can. 1257) e le persone fisiche che la gestiscono come organi o rappresentanti ${ }^{34}$; sono queste infatti che mettono in moto l'attività della persona giuridica e configurano ed esprimono la sua volontà. Questa è la relazione che più ci interessa perché sulla sua base dovrà essere costruita la responsabilità per la gestione del patrimonio della persona giuridica nei confronti di terze persone, ma anche i rispettivi diritti e doveri tra amministratore e titolare dei beni. Si potrebbe anche prospettare una qualche responsabilità della comunità che costituisce la base di molte persone giuridiche canoniche (diocesi, parrocchie, ecc.), che

\footnotetext{
${ }^{34}$ Sulle teorie organiche e rappresentative e la loro applicazione all'ordinamento canonico, cfr. J.I. Arrieta, Diritto dell'organizzazione ecclesiastica, Milano 1997, p. 106 ss.
} 
però riesce difficile da individuare in termini di esigibilità giuridica o legale ${ }^{35}$.

Lamministratore delle persone giuridiche pubbliche canoniche compie principalmente tre tipi di attività per esercitare le sue funzioni nella gestione delle risorse affidategli: garantisce la destinazione dei beni al raggiungimento delle finalità dell'ente; dirige l'amministrazione (quello che in termini di management viene chiamato governance); e sottoscrive contratti di vario genere. Tutte queste attività le compie per conto dell'ente amministrato. Molti amministratori canonici hanno anche attribuite mansioni di vigilanza sull'amministrazione delle risorse di altri enti, ma tali attività non rientrano nella loro funzione come amministratori. Il caso più rilevante è quello del vescovo diocesano, amministratore dei beni della diocesi, cui le norme del Codice attribuiscono anche la funzione di «vigilare con cura sulla amministrazione di tutti i beni appartenenti alle persone giuridiche pubbliche a lui soggette» (can. $1276 \$ 1$ ). In queste ipotesi, il vescovo, in quanto Ordinario, può incorrere in responsabilità per culpa in vigilando, ad esempio, ma tale responsabilità non proviene dal suo essere amministratore, ma rientra in altre esigenze del suo ufficio di vescovo.

Il Codice di Diritto canonico imputa la responsabilità generale per gli effetti degli atti di amministrazione dei beni temporali al titolare degli stessi, cioè alla persona giuridica ${ }^{36}$. In linea di massima, quindi, per le conseguenze degli atti di amministrazione dei beni ecclesiastici risponde l'ente cui gli stessi beni appartengono. Così si deduce, a contrario, dalla previsione di non responsabilità dell'ente in caso di atti posti invalidamente dagli amministratori (cfr. can. 1281 § 3). E così è esplicitamente stabilito nelle norme del Libro II del Codice per

\footnotetext{
${ }^{35}$ Cfr. M. D’Arienzo, Il concetto giuridico di responsabilità. Rilevanza e funzioni nel Diritto canonico, Cosenza 2012, p. 175-189, che la chiama "responsabilità partecipata". Anche noi abbiamo studiato questa responsabilità in La responsabilità canonica degli amministratori dei beni della Chiesa, Ius Ecclesiae 27 (2015) 577-594.

${ }^{36}$ Sulla validità e l'efficacia degli atti giuridici nell'ordinamento canonico, cfr. E. Baura, Parte generale del Diritto canonico. Diritto e sistema normativo, Roma 2013, p. 106-117.
} 
gli istituti di vita consacrata: «Se una persona giuridica ha contratto debiti e oneri, anche con licenza dei Superiori, è tenuta a risponderne in proprio » (can. $639 \$ 1)^{37}$.

\section{a) Responsabilità per atti invalidi}

La norma generale del Libro $\mathrm{V}$ del Codice, in effetti, prevede che «la persona giuridica non è tenuta a rispondere degli atti posti invalidamente dagli amministratori » (can. $1281 \S 3$ ). Nell’ipotesi di atto invalido posto dall'amministratore, dunque, non risponde l'ente e, anche se esplicitamente non è così stabilito, si desume che risponderà l'amministratore stesso personalmente ${ }^{38}$. La persona giuridica soltanto si assume le conseguenze di tale atto «quando e nella misura in cui ne ebbe beneficio » (can. $1281 \S 3)$.

Vi è una qualche difficoltà nella determinazione degli atti invalidi ${ }^{39}$. Non pare necessario richiamare la responsabilità nell'eventualità dell'inesistenza, del cosiddetto "atto inesistente", perché appunto l'atto non esiste per mancanza di un elemento essenziale e, quindi, non produce effetti giuridici. Tuttavia, la finzione di attività amministrativa sulla gestione delle risorse di una persona giuridica pubblica canonica che cagiona danno rientra nella responsabilità di chi la compie, come vedremo più avanti a proposito della riparazione dei danni. L'invalidità cui fa riferimento il legislatore pare riguardare piuttosto la nullità dell'atto, cioè l'amministratore pone un vero atto

\footnotetext{
${ }^{37}$ Secondo il can. $635 \$ 1$ «I beni temporali degli istituti religiosi, in quanto beni ecclesiastici, sono retti dalle disposizioni del Libro V, I beni temporali della Chiesa, a meno che non sia espressamente disposto altro». Per quanto riguarda la determinazione della responsabilità degli enti, come si vede, il testo specifico per gli istituti di vita consacrata è più "generico" e preciso di quello del Libro V.

${ }^{38}$ Bisognerà poi stabilire le altre responsabilità "personali" che possano essere coinvolte, come abbiamo accennato quando menzionavamo l'aiuto che l'economo è destinato a prestare al Vescovo diocesano, o quelle che possano essere stabilite per rappresentanti negoziali, mandatari, ecc.

${ }^{39}$ Presenta efficacemente la questione dell'esistenza, validità ed efficacia degli atti giuridici, con riferimento a diverse posizioni dottrinali, E. BAUr A, Parte generale del Diritto canonico. Diritto e sistema normativo, Roma 2013, p. 106-117.
} 
amministrativo «esistente, ma sanzionato legalmente con l'invalidità perché mancante di un elemento richiesto dal diritto positivo (...) o per vizio di un elemento essenziale dell'atto (...). Diversamente da quanto succede nell'atto inesistente, affinché un atto sia nullo deve essere così stabilito expresse da una legge (can. 10) ${ }^{40}$.

Il can. 10 definisce "irritanti" le leggi «con le quali si stabilisce espressamente che l'atto è nullo». In materia di gestione dei beni ecclesiastici, il legislatore stabilisce espressamente

- la nullità dell'alienazione di beni appartenenti al patrimonio stabile senza le dovute licenze (cfr. can. 1291) ${ }^{41}$, anche per gli istituti di vita consacrata (cfr. can. $638 \$ 3$ ) ${ }^{42}$. Nel caso specifico delle diocesi e delle strutture ad esse assimilate (cfr. can. 368), invece, il can. $1292 \$ 1$ prevede che il vescovo ottenga il consenso del Consiglio diocesano per gli affari economici e del Collegio dei consultori, ma non richiede una licenza in senso proprio, quando l'alienazione riguarda un bene stimato in un valore che è al di sotto della quantità massima stabilita dalla Conferenza episcopale (cfr. can. $1292 \$ 2$ ). Il tentativo di alienazione fa sorgere anche responsabilità penale, in quanto costituisce delitto a norma del can. 1377: «Chi senza la debita licenza aliena beni ecclesiastici sia punito con giusta pena »;

- la nullità degli atti posti in «qualunque altro negozio che intacchi il patrimonio della persona giuridica peggiorandone la condizione » (can. 1295) senza gli stessi requisiti previsti dai canoni 1291-1294 per l'alienazione in senso proprio, cioè l'alienazione

\footnotetext{
${ }^{40}$ E. Baura, Parte generale del Diritto canonico, cit., p. 110.

${ }^{41}$ «Per alienare validamente [valide] i beni che costituiscono per legittima assegnazione il patrimonio stabile di una persona giuridica pubblica, e il cui valore ecceda la somma fissata dal diritto, si richiede la licenza dell'autorità competente a norma del diritto». Riportiamo il testo del canone perché il lettore possa valutare meglio le parole adoperate per stabilire la nullità nelle diverse fattispecie.

${ }^{42}$ «Per la validità [validitatem] dell'alienazione, e di qualunque negozio da cui la situazione patrimoniale della persona giuridica potrebbe subire detrimento, si richiede la licenza scritta rilasciata dal Superiore competente con il consenso del suo consiglio. (...)».
} 
che riguarda i beni del patrimonio stabile, secondo il primo dei canoni richiamati. In riferimento agli istituti di vita consacrata, il can. $638 \$ 3$ stabilisce la nullità di «qualunque negozio da cui la situazione patrimoniale della persona giuridica potrebbe subire detrimento » in assenza degli stessi requisiti previsti per l'alienazione;

- la nullità degli atti di amministrazione straordinaria posti senza il previo «permesso scritto dell'Ordinario » (can. $1281 \$ 1)^{43}$, che vale anche per gli istituti di vita consacrata, a norma del can. 638 $\$ 1,{ }^{44}$ e probabilmente anche per le diocesi, anche se in questa fattispecie specifica il can. 1277 non lo stabilisce espressamente e si limita a richiedere il consenso del consiglio per gli affari economici e del collegio dei consultori ${ }^{45}$. A nostro avviso, sarebbe poco ragionevole che l'invalidità degli atti di straordinaria amministrazione fosse stabilita per tutti gli amministratori tranne i vescovi ${ }^{46}$. Infatti, il can. 127 prevede i modi in cui la

${ }^{43}$ «(...) gli amministratori pongono invalidamente [invalide] atti che oltrepassano i limiti e le modalità dell'amministrazione ordinaria, a meno che non abbiano ottenuto prima permesso scritto dall'Ordinario». La denominazione "atti di amministrazione straordinaria" è adoperata dagli autori per riferirsi a questi atti.

${ }^{44}$ «Spetta al diritto proprio determinare, entro l'ambito del diritto universale, quali sono gli atti che eccedono il limite e le modalità dell'amministrazione ordinaria, e stabilire ciò che è necessario per porre validamente [valide] gli atti di amministrazione straordinaria».

${ }^{45}$ «Il Vescovo diocesano (...) ha (...) bisogno del consenso del medesimo consiglio [per gli affari economici] ed anche del collegio dei consultori, oltre che nei casi specificamente espressi nel diritto universale o nelle tavole di fondazione, per porre atti di amministrazione straordinaria. (...)».

${ }^{46}$ Alcune Conferenze episcopali, nel determinare gli atti di amministrazione straordinaria nella gestione della diocesi, hanno stabilito che tali atti siano invalidi in assenza dei requisiti stabiliti dal can. 1277. In tali casi, la legislazione particolare impone espressamente alle diocesi del territorio della Conferenza la sanzione della nullità di detti atti. Ad esempio, la Conferenza episcopale degli Stati Uniti di America parla esplicitamente di validità degli atti: «the United States Conference of Catholic Bishops, in accord with the norm of canon 1277, decrees that the following are to be considered acts of extraordinary administration, the canonical validity of which requires the diocesan bishop to obtain the consent of the diocesan 
mancanza di consenso di un collegio o gruppo a cui deve essere chiesto comporta l'invalidità dell'atto;

- la nullità degli «atti di amministrazione, che, attesa la situazione economica della diocesi, sono di maggior importanza» (can. 1277) posti dal Vescovo senza «udire il consiglio per gli affari economici e il collegio dei consultori» (ibid.) o il parere di tutti i loro componenti, a norma del can. $127 \$ 1$ : «Quando dal diritto è stabilito che il Superiore per porre gli atti necessiti del consenso o del consiglio di un collegio o di un gruppo di persone, il collegio o il gruppo deve essere convocato a norma del can. 166, a meno che, quando si tratti di richiedere soltanto il consiglio, non sia stato disposto altrimenti dal diritto particolare o proprio; perché poi l'atto valga [valeant] si richiede che sia ottenuto il consenso della maggioranza assoluta di quelli che sono presenti o richiesto il consiglio di tutti».

b) Responsabilità per atti illegittimi

La persona giuridica risponde, invece, «degli atti posti validamente ma illegittimamente dagli amministratori salva l'azione o il ricorso da parte sua contro gli amministratori che le abbiano arrecato danni» (can. $1281 \$ 3$ ). Con questa previsione, il legislatore riporta gli atti illegittimi al criterio di responsabilità generale della persona giuridica.

Non è facile individuare l'illegittimità di un atto valido. De Paolis afferma che «l'atto è soltanto illegittimo, se ha violato delle norme solo proibenti o imperative» ${ }^{47}$. In questo senso, sembra potersi ritenere valido ma illegittimo l'atto di alienazione compiuto senza "giusta causa" o senza procedere alla "stima della cosa da alienare" o "senza le altre cautele prescritte" richieste dal can. 1293, ma con le licenze richieste dal can. 1292. In effetti, il testo legale indica questi requisiti

finance council and the college of consultors» (USCCB, Complementary Norms, in www.usccb.org/beliefs-and-teachings/what-we-believe/canon-law/complementary-norms/index.cfm).

${ }^{47}$ V. De PaOlis, I beni temporali della Chiesa, Bologna 1995, p. 165. 
senza esprimere la sanzione di nullità ${ }^{48}$. Il regime di responsabilità risultante da questa nostra supposizione soddisfa l'esigenza di giustizia di chi dovesse essere danneggiato da un tale atto: gli si può richiedere in alcune circostanze (ad esempio, in Italia per legislazione di origine pattizia tra lo Stato e la Santa Sede) di sapere che l'amministratore non può alienare senza licenza, ma non di accertarsi che l'amministratore stesso abbia valutato l'esistenza o meno di una giusta causa, abbia provveduto alla stima del bene, ecc. Se l'amministratore si presenta davanti a un terzo dotato delle licenze previste, egli può ritenere che agisca legittimamente; se poi si dimostrasse che non è stato così, l'illegittimità non dovrebbe intaccare la validità dell'atto.

Un ragionamento simile varrebbe nel caso di alienazione compiuta dall'amministratore «a prezzo minore di quello indicato nella stima» (can. $1294 \$ 1$ ): il legislatore non sancisce di nullità tale negozio, anche se comporta un'azione illegittima da parte dell'amministratore. La persona giuridica non potrà chiedere al compratore di integrare il pagamento fatto fino a raggiungere la stima che l'amministratore non ha rispettato, ma potrà agire contro l'amministratore stesso per chiedere la differenza e gli altri eventuali danni che l'affare possa aver provocato. Sembra giusto che in queste ipotesi risponda la persona giuridica davanti al terzo, senza impedire di risanare il danno arrecato dall'amministratore mediante l'azione o il ricorso opportuni.

Valgano, quindi, per questa ipotesi di responsabilità per atti illegittimi dell'amministratore gli esempi fatti. In realtà, che l'atto illegittimo ma valido comporti la responsabilità in primo luogo della persona giuridica, come dicevamo, riporta al criterio generale per il quale ogni soggetto, anche le persone giuridiche, risponde per gli atti da lui compiuti. E per esemplificare possibilità di agire illegittimo dell'amministratore si potrebbe andare a ripercorrere tutti

\footnotetext{
${ }^{48}$ Can. 1293 - \$1. Per l'alienazione dei beni il cui valore eccede la somma stabilita, si richiede inoltre: 1) una giusta causa, quale la necessità urgente, l'utilità palese, la pietà, la carità o altra grave ragione pastorale; 2) la stima della cosa da alienare fatta da periti per iscritto. $\$ 2$. Si osservino inoltre le altre cautele prescritte dall'autorità legittima per evitare danni alla Chiesa.
} 
i suoi obblighi e ipotizzare casi di inadempimento, oppure immaginare l'inadempienza del criterio comportamentale fondamentale espresso dal can. 1284 con la figura della "diligenza di un buon padre di famiglia" ${ }^{39}$.

Tutte le ipotesi di responsabilità previste dal legislatore canonico si esauriscono nella persona giuridica per conto della quale si è agito. Il responsabile per la gestione delle risorse di un ente è l'ente stesso oppure, in casi di invalidità degli atti posti, l'amministratore. In nessuna fattispecie è chiamato a rispondere il superiore dell'ente amministrato, a meno che non abbia mancato al suo dovere di vigilanza o di controllo, ma qui non si tratterebbe di responsabilità nella gestione delle risorse bensì di responsabilità nell'azione di governo. Questo è il corollario dell'applicazione del principio di autonomia degli enti all'amministrazione delle risorse.

\section{Responsabilità giuridica dei singoli fedeli}

Per completare il quadro della responsabilità nell'amministrazione delle risorse conviene accennare ancora a qualche peculiarità prevista dalla legge canonica. Il legislatore canonico non stabilisce la responsabilità contrattuale $\mathrm{o}$ extra contrattuale che possano contrarre i singoli fedeli nei propri affari, anche nell'eventualità in cui il fedele coinvolto in una determinata fattispecie fosse pure amministratore di una persona giuridica pubblica. Certamente, potrebbe essere interessante studiare le responsabilità cui vengono incontro i fedeli in diverse ipotesi, ma non riguarderebbero l'amministrazione dei beni ecclesiastici né l'autonomia degli enti, che sono l'oggetto del nostro studio. Per lo stesso motivo abbiamo escluso la trattazione della responsabilità degli amministratori di persone giuridiche private e degli enti senza personalità.

\footnotetext{
${ }^{49}$ Si pensi ad un amministratore che compra qualcosa di inutile per la persona giuridica amministrata. Il venditore consegnerà la cosa e la persona giuridica dovrà pagarne il prezzo, ma l'agire dell'amministratore sarà stato illegittimo e, quindi, secondo quanto stabilito dal can. $1281 \$ 3$, la persona giuridica compratrice potrebbe agire giudizialmente contro l'amministratore che ha arrecato il danno.
} 
Tuttavia, vi sono due ipotesi in cui la responsabilità dei fedeli potrebbe rilevare per la comprensione generale della materia dei beni temporali nella Chiesa, l'una oggettiva e l'altra soggettiva. L'ipotesi oggettiva riguarda il regime "speciale" delle cose sacre, quella soggettiva si riferisce alla peculiare condizione canonica di alcuni fedeli, concretamente dei religiosi o dei fedeli appartenenti ad un istituto di vita consacrata.

«Le cose sacre, quelle cioè che sono state destinate al culto divino con la dedicazione o la benedizione» (can. 1171), hanno un regime legale specifico che comporta che non possano essere «adoperate per usi profani o impropri, anche se sono in possesso ${ }^{50}$ di privati» (ibid.) $)^{51}$. L'amministratore o il proprietario di tali "cose" risponde davanti alla Chiesa della destinazione della cosa al culto e anche dell'adempimento delle prescrizioni legittime sull'uso di tali "cose". Si applica qui il criterio generale della responsabilità nell'ordinamento canonico: risponde chi agisce, anche se si tratta di una persona giuridica. Nel caso delle cose e i luoghi sacri, l'amministratore risponde anche della loro destinazione al culto pubblico e dell'uso adeguato alla loro natura. Ma chiunque si trovi a gestire una cosa sacra si rende responsabile della sua destinazione al culto e del suo uso decoroso. In questa fattispecie, l'ordinamento stabilisce una responsabilità che poggia sulla natura della cosa e non cambia per considerazione al soggetto che la gestisce.

Per l'ipotesi soggettiva, la condizione canonica e sociale dei religiosi fa sì che il legislatore abbia previsioni specifiche sulla loro responsabilità nella gestione dei beni temporali ${ }^{52}$. Concretamente, il can. 639 prevede che un religioso possa agire sui propri beni, nel qual

\footnotetext{
${ }^{50}$ L'originale latino riporta dominio, in riferimento quindi alla proprietà, dove la traduzione parla di "possesso".

${ }^{51}$ Per gli effetti che adesso ci interessano vanno ricomprese nella nozione di cose sacre anche i luoghi sacri: «Sono sacri quei luoghi che vengono destinati al culto divino o alla sepoltura dei fedeli mediante la dedicazione o la benedizione, a ciò prescritti dai libri liturgici» (can. 1205).

${ }^{52}$ L'ordinamento canonico stabilisce l'“autonomia” degli Istituti di vita consacrata, con effetti giuridici specifici (cfr. Y. Sugawara, Autonomía de los IVC, in:
} 
caso risponde personalmente $e^{53}$ o che possa concludere un negozio per l'istituto, sia per mandato dei superiori, e in questa fattispecie risponde l'istituto ${ }^{54}$, sia senza alcuna licenza, e allora risponde il religioso stesso ${ }^{55}$. Queste norme tengono conto della peculiare condizione del religioso, che potrebbe portare i terzi a pensare che sia legittimo rappresentante dell'istituto, e tendono a proteggere più l'istituto che il terzo che negozia con il religioso. La norma rientra nella logica abituale per la quale chi entra in affari con una persona giuridica deve accertarsi che chi la rappresenta sia provvisto del mandato necessario o svolga un ufficio che gli consenta di agire per conto di essa. Il canone aggiunge una clausola che conferisce azione contro chiunque abbia tratto vantaggio dal negozio ${ }^{56}$.

In materia di specificità dei religiosi e degli istituti di vita consacrata, il legislatore ha previsto per loro anche una "responsabilità esemplare", per chiamarla in qualche modo, un richiamo alla testimonianza personale, mediante il voto di povertà (cfr. can. 600), e anche collettiva di "carità e di povertà" e di preoccupazione per i bisognosi (cfr. can. 640). Certamente, questa "responsabilità" rimane più morale che giuridica, nel senso che è difficile determinare quale sia la misura del dovuto nelle singole fattispecie. Tuttavia, l'amministrazione dei beni ecclesiastici di queste persone giuridiche pubbliche nella Chiesa non può prescindere dal richiamo alla esemplarità della povertà.

Diccionario General de Derecho Canónico, vol. I, cit., p. 579-582, con indicazioni bibliografiche).

${ }^{53}$ «Se un religioso con licenza del Superiore ha contratto debiti e oneri sui beni propri, ne deve rispondere personalmente (...)» (can. $639 \$ 2)$.

${ }^{54}$ «(...) se invece per mandato del Superiore [un religioso] ha concluso affari dell’istituto, è l’istituto che ne deve rispondere» (can. $639 \$ 2$ ).

55 «Se un religioso li ha contratti [gli affari] senza alcuna licenza del Superiore, è lui stesso, e non la persona giuridica, a doverne rispondere» (can. 639 \$3).

56 «Rimanga fermo tuttavia che si può sempre intentare un'azione contro colui il cui patrimonio si è in qualche misura avvantaggiato in seguito a quel contratto» (can. $639 \$ 4$ ). 


\section{La riparazione dei danni}

La giustizia implica una costante tensione verso l'assegnazione a ciascuno di ciò che gli spetta. Quando, in circostanze determinate, quella corrispondenza tra le "cose" e le "persone" è stata violata in qualsiasi modo, la giustizia esige riparazione. La riparazione dei danni arrecati è quindi necessaria per il ripristino della giustizia in ogni società. Tutti gli ordinamenti legali, in un modo o nell'altro, prevedono i modi per la riparazione dei danni.

Il legislatore canonico ha prescritto che «chiunque illegittimamente con un atto giuridico, anzi con qualsiasi altro atto posto con dolo o con colpa, arreca danno ad un altro, è tenuto all'obbligo di riparare il danno arrecato» (can. 128). La prospettiva assunta in questa norma è quella soggettiva: non si stabilisce cosa occorra fare ma chi deve farlo (chiunque arreca danno). Ma si possono trarre delle indicazioni sul modo di riparare i danni da alcune proposte fatte dagli autori. Ad esempio, relativamente alla tipologia dei danni risarcibili, Regojo Bacardí ritiene che la Chiesa deve concedere e concede la riparazione non soltanto per danni economici, ma anche, forse principalmente, per danni morali e spirituali ${ }^{57}$. Pree aggiunge che la forma e la modalità del risarcimento devono corrispondere al genere dell'atto lesivo e del danno, e devono anche tener conto delle peculiarità della Chiesa, della sua missione e del suo fine ${ }^{58}$.

Inoltre, abitualmente si distingue tra la reintegrazione (riparazione in forma specifica; rimozione del danno recato) e il risarcimento (riparazione in forma generica o pecuniaria) ${ }^{59}$. Ad ogni modo, il legislatore prevede che chiunque causa un danno "con qualsiasi atto"

\footnotetext{
${ }^{57}$ Cfr. G. Regojo Bacardí, Pautas para una concepción canónica del resarcimiento de daños, Fidelium Iura 4 (1994) 107-162; M. Thériault, can. 128, in: Comentario exegético al Código de Derecho canónico, vol. 1, Pamplona 1996, p. 835-836..

${ }^{58}$ Cfr. H. Pree, can. 128, in: Münsterischer Kommentar zum Codex Iuris Canonici, I, Essen 1998.

${ }^{59}$ Cfr. F. D’Ostilio, La responsabilità per atto illecito della pubblica amministrazione nel diritto canonico, Roma 1966, p. 114-120
} 
deve ripararlo ${ }^{60}$. La richiesta del dolo o la colpa aggiunge complessità che in questo momento non ci interessano ${ }^{61}$.

Per la riparazione dei danni in materia contrattuale e di pagamenti, a norma del can. 1290 occorrerà rifarsi alla legislazione civile vigente nel territorio, come abbiamo già accennato. Tale legislazione comprende anche le norme internazionali vigenti nel territorio. E questi modi e misure di riparazione previsti dalle norme civili saranno applicati anche dal giudice ecclesiastico con gli stessi effetti. Vale a dire, in una causa canonica per riparazione dei danni causati da un contratto o un pagamento, a meno che il legislatore non abbia previsioni concrete nelle norme canoniche (o che la legge civile richiamata possa essere ritenuta contraria al diritto divino, cosa poco probabile), il giudice ecclesiastico applicherà la legge vigente nel territorio che viene canonizzata per quella fattispecie ${ }^{62}$.

Il diritto dei religiosi, come già accennato, nelle ipotesi di responsabilità derivante da contratto, aggiunge alla generale possibilità di chiedere la riparazione dei danni, a norma del can. 128, «un'azione contro colui il cui patrimonio si è in qualche misura avvantaggiato in seguito a quel contratto $»(\text { can. } 639 \$ 4)^{63}$.

${ }^{60}$ Cfr. G.P. Montini, Resarcimiento de daños, in: Diccionario General de Derecho Canónico, vol. VI, cit., p. 949-952.

${ }^{61} \mathrm{Si}$ pensi all'imputabilità e altri istituti simili. Vi sono alcuni studi recenti che trattano questi argomenti, ad es., M. D'Arienzo, L'obbligo di riparazione del danno in diritto canonico. Percorsi di ricerca, Cosenza 2013

${ }^{62}$ Non possiamo fermarci adesso ad analizzare la tecnica del rinvio normativo, frequente nel Diritto internazionale privato e anche nel Diritto canonico. Per studi più approfonditi, cfr. P. Ciprottr, Canonizzazione delle leggi civili, in: Digesto delle discipline pubblicistiche, $4^{\text {a }}$ ed., Torino 1988; J. Miñambres, Análisis de la técnica de la remisión a otros ordenamientos jurídicos en el Código de 1983, Ius canonicum 32 (1992) 713-749; C. Minelli, La canonizzazione delle leggi civili e la codificazione postconciliare. Per un approccio canonistico al tema dei rinvii tra ordinamenti, Periodica 85 (1996) 445-487; G. Boni, La rilevanza del diritto dello Stato nell'ordinamento canonico. In particolare la canonizatio legum civilium, Milano 1998.

${ }^{63}$ Come abbiamo già detto nel testo, qui si fa riferimento principalmente alla gestione dei beni propri dei religiosi, che in questo momento non ci interessano, 


\section{Conclusioni}

Per concludere questa breve presentazione della responsabilità nell'amministrazione dei beni ecclesiastici, se mettiamo insieme i rilievi fatti sul principio di autonomia nell'organizzazione e quelli riguardanti la responsabilità degli enti, viene fuori un impianto legale secondo il quale nel diritto canonico ogni persona, fisica o giuridica, risponde per gli atti propri intra vires, nei limiti cioè delle proprie risorse patrimoniali. Questo ci sembra il principio che regge tutta la struttura della responsabilità nel Diritto canonico: per le conseguenze degli atti, risponde chi agisce.

Logicamente, per evitare ingiustizie, il legislatore ha pensato meccanismi che consentono di scaricare parte della responsabilità sul beneficiario dell'atto anche quando esso fosse invalido, come abbiamo visto. Ma tale previsione non inficia il principio per cui chi agisce risponde per le conseguenze dell'atto.

Il richiamo al principio di autonomia nell'organizzazione ecclesiastica consente di cogliere il limite delle risorse messe in gioco con la propria azione: ogni soggetto risponde con le risorse che sono nella sua titolarità. Anche se a prima vista, questa conclusione può risultare ovvia, la peculiare configurazione della comunione della Chiesa potrebbe farla perdere di vista. È frequente che un ente gerarchicamente superiore a quello che ha agito venga incontro alle conseguenze degli atti posti nella gestione delle risorse di quello subordinato. È probabilmente non soltanto buono ma necessario che ciò accada. Basti pensare al peculiare rapporto fra diocesi e parrocchie, nel quale emerge, da una parte l'interesse della diocesi perché la parrocchia svolga al meglio le proprie attività, e dall'altra la dipendenza pastorale della parrocchia dalla diocesi. Ma questi stretti rapporti fra gli enti

ma anche a quella dei beni dell'istituto in quanto si potrebbe ritenere che il religioso rappresenti in qualche modo l'istituto. Un collegamento simile potrebbe essere stabilito tra i chierici secolari e le strutture di incardinazione, anche se qui la responsabilità nella gestione delle risorse ecclesiastiche dovrebbe essere accertata da chi ha su di esse il compito di vigilanza. Cfr. J. Ferrer, La responsabilidad civil de la diócesis por los actos de sus clérigos, Ius canonicum 45 (2005) 557-608. 
non implicano minore autonomia nella gestione delle risorse di ciascuno, ma piuttosto obblighi di vigilanza e di rendicontazione che, in senso proprio, esulano dall'amministrazione dei beni ecclesiastici. Per questo motivo, alcune soluzioni addotte per il riconoscimento degli enti ecclesiastici in taluni ordinamenti pongono problemi proprio dal punto di vista dell'autonomia, che tendono a riflettersi anche sulla responsabilità ${ }^{64}$.

Migliorare la giustizia nell'uso delle risorse ecclesiastiche è un'esigenza che non può prescindere da una adeguata organizzazione (autonomia) e dalle previsioni normative per eventuali anomalie (leggi sulla responsabilità). Dal punto di vista dell'organizzazione, appare quanto mai opportuno garantire con tutti i mezzi a disposizione l'autonomia degli enti, in modo da distinguere bene tra amministrazione e vigilanza. Dal punto di vista delle norme che fissano le responsabilità per gli atti di amministrazione, forse occorrerebbe proteggere meglio le "apparenze", tradizionale principio in questo campo per cui non si ammette che un terzo venga danneggiato da chi apparentemente agisce in un determinato ruolo (si pensi, ad esempio, alle fattispecie che contemplano l'agire dei religiosi nei confronti degli istituti o dei sacerdoti in relazione alle diocesi). La combinazione di entrambe le esigenze aiuta a una migliore delimitazione del perimetro della responsabilità, e garantisce una giustizia più aderente alla realtà.

\footnotetext{
${ }^{64}$ Il sistema di incorporation di alcune diocesi degli Stati Uniti come corporation sole, per il quale il vescovo diocesano diviene titolare per la legge civile, in quanto vescovo, di tutte le risorse ecclesiastiche presenti nella propria diocesi, con l'onere conseguente di amministrarle, è difficilmente compatibile con la necessaria autonomia degli enti canonici e comporta una responsabilità in capo al vescovo che non si corrisponde con quella pensata dal legislatore canonico. In questi sistemi, o in altri simili, per le autorità dello Stato il vescovo risulta amministratore di tutti i beni ecclesiastici presenti nella sua diocesi e non "superiore", come prevede l'impianto della legge canonica.
} 


\section{Autonomy and responsibility in the management of the resources of the Church}

To determine the responsibility for juridical acts, one must first establish the limits of the agent's autonomy. This article studies the limitations of the autonomy of juridical persons, administrators, and other faithful in the management of the resources entrusted to the Church's institutions.

PAROLE CHIAVE: autonomia, responsabilità giuridica, amministrazione, vigilanza

Key Words: Autonomy, Juridical Responsibility, Administration, Vigilance

\section{Nota o Autorze:}

Prof. Jesús Miñambres - sacerdote, professore ordinario di diritto patrimoniale canonico presso la Facoltà di Diritto Canonico della Pontificia Università della Santa Croce; decano della medesima Facoltà; Socio della Consociatio internationalis studio iuris canonici promovendo e dell'Associazione canonistica italiana; consultore della Congregazione per il Clero. 